\title{
ON THE THEORY OF SUM-EQUATIONS
}

\section{M. SHEFFER}

1. Introduction. A system of linear equations is called a sumequation system if it has the form

$$
a_{n 0} x_{n}+a_{n 1} x_{n+1}+\cdots=c_{n} \quad(n=0,1, \cdots),
$$

with $a_{n 0} \neq 0$. All quantities are assumed to be complex numbers. Let $\left\{A_{n}(t)\right\}$ be defined by

$$
A_{n}(t)=\sum_{s=0}^{\infty} a_{n s} t^{s} \quad(n=0,1, \cdots),
$$

and define the type of a sequence $\left\{y_{n}\right\}$ as the number $\left(\left(y_{n}\right)\right)$ given by

$$
\left(\left(y_{n}\right)\right) \equiv \limsup _{n \rightarrow \infty}\left|y_{n}\right|^{1 / n} \text {. }
$$

Suppose the functions $\left\{A_{n}(t)\right\}$ are analytic in $|t|<q$, and that $\left(\left(c_{n}\right)\right)=\delta<q$, so that the function

$$
C(t)=\sum_{0}^{\infty} c_{n} t^{n}
$$

has the radius of convergence $1 / \delta$.

Let functions $\left\{H_{k, j}(t)\right\}(j=0,1, \cdots, k-1 ; k=0,1, \cdots)$ be defined by

$$
H_{k, j}(t)=c_{j}+c_{j+k} t^{k}+c_{j+2 k} t^{2 k}+\cdots,
$$

and let

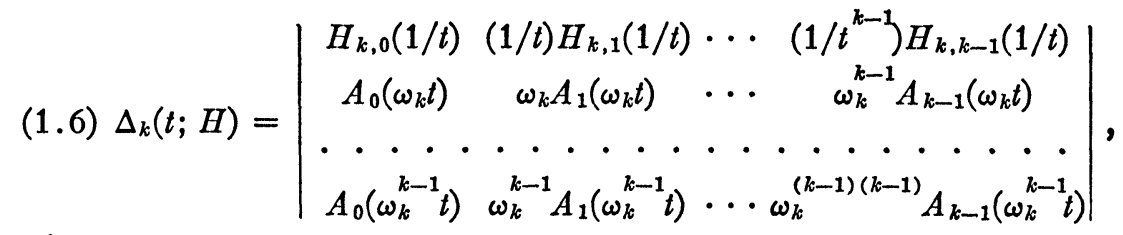

where

$$
\omega_{k}=e^{2 \pi i / k} \quad\left[i=(-1)^{1 / 2}\right] .
$$

The functions $H_{k, j}(t)$ are analytic in $|t|<1 / \delta$, the function $\Delta_{k}(t)$ given by

Presented to the Society, April 17, 1948; received by the editors June 28, 1948. 


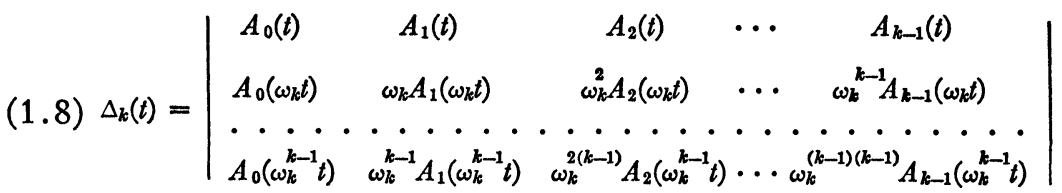

in $|t|<q$, and the function $\Delta_{k}(t ; H)$ in the ring $\delta<|t|<q$.

The present article has two principal aims. One is to establish the following result on sum-equations:

THEOREM 1.1. Let the functions $\left\{A_{n}(t)\right\}$ be analytic in $|t|<q$, with $A_{n}(0) \neq 0$, and let $\left(\left(c_{n}\right)\right)=\delta<q$. If there exists an increasing sequence of integers $k_{s}(s=1,2, \cdots)$ such that

$$
\lim _{s=\infty} k_{s} \Delta_{k_{s}}(t ; H) / \Delta_{k_{s}}(t)=\Delta^{*}(t),
$$

the convergence being uniform in a ring $R: q_{1}<|t|<q_{2}$, with $\delta<q_{2} \leqq q$, then

$$
x_{n}=\frac{1}{2 \pi i} \int_{\gamma} t^{n-1} \Delta^{*}(t) d t
$$

is a solution of system (1.1), where $\gamma$ is a rectifiable simple closed curve surrounding $t=0$ and lying in the ring $R$. Moreover, $\left(\left(x_{n}\right)\right) \leqq d$ where $d$ is the maximum distance from $\gamma$ to the origin.

This theorem will be obtained in $\$ 3$ as the result of a limiting process based on the solution of associated $k$-periodic systems of equations. The second aim is to obtain solutions of homogeneous and nonhomogeneous $k$-periodic systems, in closed form, as contour integrals. This is done in $\S \S 2$ and 4.

2. Nonhomogeneous $k$-periodic system. Sum-systems that are $k$-periodic (with or without perturbation terms) were treated elsewhere. ${ }^{1}$ There the existence theorems did not give solutions in closed form. We now obtain closed solutions. System

$$
a_{n 0} x_{n}+a_{n 1} x_{n+1}+\cdots=c_{n} \quad(n=0,1, \cdots)
$$

is $k$-periodic if

$$
A_{k n+p}(t)=A_{p}(t) \quad(p=0,1, \cdots, k-1 ; n=0,1, \cdots),
$$

where the functions $\left\{A_{n}(t)\right\}$ are given by (1.2). We suppose here that

1 I. M. Sheffer, On k-periodic systems of linear equations, Trans. Amer. Math. Soc. vol. 63 (1948) pp. 244-313. We shall refer to this article as Tr. 
each $A_{n}(t)$ is analytic in $|t| \leqq q$ (so that $q$ can be replaced by $q+\epsilon$ for sufficiently small positive $\epsilon)$, and that $\left(\left(c_{n}\right)\right)=\delta \leqq q$.

Let $\gamma$ be the circle $|t|=q^{\prime}$, where $\delta<q^{\prime}$, and where $q^{\prime}$ is chosen close enough to $\delta$ so that the functions $\left\{A_{n}(t)\right\}$ are regular ${ }^{2}$ in $|t| \leqq q^{\prime}$ and also so that the function $\Delta_{k}(t)$ given by (1.8) has no zeros on $\gamma$. Define the sequence $z_{n}^{(j)}$ by

$$
z_{n}^{(j)}=\frac{1}{2 \pi i} \int_{\gamma} \frac{t^{n+\sigma}}{\Delta_{k}(t)} \cdot \Delta_{k ; 0, j}(t) H_{k, \nu}\left(\frac{1}{t}\right) d t,
$$

where $H_{k, v}$ is given by (1.5) and $\Delta_{k ; r, j}(t)$ is the cofactor of the element in the rth row and jth column in $\Delta_{k}(t)$. Moreover, $\sigma$ and $\nu$ are (as yet undetermined) integers whose value will be made to depend on $j$.

Substitute the sequence $z_{n}^{(j)}$ into the $(k s+r)$ th equation of $(2.1)$. By uniform convergence, and the use of (2.2),

$$
\begin{aligned}
A_{k s+r}\left[\left\{z_{n}^{(j)}\right\}\right] & \equiv \sum_{n=0}^{\infty} a_{k s+r, n} z_{k s+r+n}^{(j)} \\
& =\frac{1}{2 \pi i} \int_{\gamma} \frac{t^{k s+r+\sigma}}{\Delta_{k}(t)} A_{r}(t) \Delta_{k ; 0, j}(t) H_{k, \nu}\left(\frac{1}{t}\right) d t .
\end{aligned}
$$

Make the substitution $t=\omega_{k}^{\lambda} u$, where $\omega_{k}$ is given by (1.7) and $\lambda$ is an integer. As $t$ traverses $\gamma$, so does $u$, and on using the relations ${ }^{3}$

$$
\begin{aligned}
\Delta_{k}\left(\hat{\omega}_{k} t\right) & =\Delta_{k}(t), \\
H_{k, \nu}\left(\omega_{k} t\right) & =H_{k, \nu}(t),
\end{aligned}
$$

we obtain

$$
\begin{aligned}
A_{k s+r}\left[\left\{z_{n}^{(j)}\right\}\right]= & \frac{1}{2 \pi i} \int_{\gamma} \frac{u^{k s+r+\sigma}}{\Delta_{k}(u)} \cdot H_{k, \nu}\left(\frac{1}{u}\right) \omega_{k}^{\lambda(r+\sigma+1)} \\
& \cdot A_{r}\left(\omega_{k}^{\lambda} u\right) \cdot \Delta_{k ; 0, j}\left(\omega_{k}^{\lambda} u\right) d u .
\end{aligned}
$$

Now sum over $\lambda=0,1, \cdots, k-1$ and divide by $k$ :

$$
\begin{aligned}
A_{k s+r}\left[\left\{z_{n}^{(j)}\right\}\right]= & \frac{1}{2 \pi k i} \int_{\gamma} \frac{u^{k s+r+\sigma}}{\Delta_{k}(u)} \\
& \cdot H_{k, \nu}\left(\frac{1}{u}\right)\left\{\sum_{\lambda=0}^{k-1} \omega_{k}^{\lambda(r+\sigma+1)} A_{r}\left(\omega_{k}^{\lambda} u\right) \Delta_{k ; 0, j}\left(\omega_{k}^{\lambda} u\right)\right\} d u .
\end{aligned}
$$

2 This requirement is especially needed when $\delta=q$.

${ }^{3}$ (2.5) appears as Theorem 2.2 in $\mathrm{Tr}$. (loc. cit.); and (2.6) is an immediate consequence of (1.5). 
From Lemma 3.2 of Tr. (loc. cit) we find that

$$
\Delta_{k ; 0, j}^{\lambda}\left(\omega_{k} t\right)=\omega_{k}^{\lambda j} \Delta_{k ; \lambda, j}(t) ;
$$

so

$$
\begin{aligned}
& A_{k s+r}\left[\left\{z_{n}^{(j)}\right\}\right]=\frac{1}{2 \pi k i} \int_{\gamma} \frac{u^{k s+r+\sigma}}{\Delta_{k}(u)} \\
& \cdot H_{k, \nu}\left(\frac{1}{u}\right)\left\{\sum_{\lambda=0}^{k-1} \omega_{k}^{\lambda(j+\sigma+1)}\left[\omega_{k}^{\lambda r} A_{r}\left(\omega_{k}^{\lambda} u\right) \Delta_{k ; \lambda, j}(u)\right]\right\} d u .
\end{aligned}
$$

But from the definition of $\Delta_{k ; \lambda, j}$ as a cofactor in the determinant $\Delta_{k}(t)$, we have

$$
\sum_{\lambda=0}^{k-1} \omega_{k}^{\lambda r} A_{r}\left(\omega_{k}^{\lambda} u\right) \Delta_{k ; \lambda, j}(u)=\left\{\begin{array}{ccc}
\Delta_{k}(u) & \text { if } & \jmath=r \\
0 & \text { if } & j \neq r .
\end{array}\right.
$$

Consequently, if we choose $\sigma=-j-1$, as we now do, then the brace in (2.10) has the value $\Delta_{k}(u)$ or 0 according as $j=r$ or $j \neq r$.

Thus, for $r, j=0,1, \cdots, k-1 ; s=0,1, \cdots$,

$$
A_{k s+r}\left[\left\{z_{n}^{(j)}\right\}\right]=\left\{\begin{array}{cl}
\frac{1}{2 \pi k i} \int_{\gamma} u^{k s-1} H_{k, \nu}\left(\frac{1}{u}\right) d u, & j=r ; \\
0, & j \neq r .
\end{array}\right.
$$

Now choose $\nu=j$, and define $\left\{y_{n}\right\}$ by

$$
y_{n}=\sum_{j=0}^{k-1} z_{n}^{(j)}
$$

so that

$$
y_{n}=\frac{1}{2 \pi i} \int_{\gamma} \frac{t^{n-1}}{\Delta_{k}(t)}\left\{\sum_{j=0}^{k-1} t^{-i} \Delta_{k ; 0, j}(t) H_{k, j}\left(\frac{1}{t}\right)\right\} d t .
$$

Then from (2.12) we see that

$$
\begin{aligned}
A_{k s+r}\left[\left\{y_{n}\right\}\right] & =\frac{1}{2 \pi k i} \int_{\gamma} u^{k s-1} H_{k, r}\left(\frac{1}{u}\right) d u \\
& =\frac{1}{k}\left[\text { coefficient of } u^{-k s} \text { in } H_{k, r}\left(\frac{1}{u}\right)\right] \\
& =\frac{1}{k} c_{k s+r},
\end{aligned}
$$

so the following result has been established: 
THEOREM 2.1. Let system (2.1) be k-periodic, and let the functions $\left\{A_{n}(t)\right\}$ be analytic in $|t| \leqq q$, with $A_{n}(0) \neq 0$. Then for each sequence $\left\{c_{n}\right\}$ of type $\delta \leqq q,(2.1)$ has the solution

$$
x_{n}=k y_{n}
$$

where $\left\{y_{n}\right\}$ is given by (2.14), the contour $\gamma$ being the circle $|t|=q^{\prime}$, where $q^{\prime}$ is larger than $\delta$ but smaller than the minimum radius of convergence of the sequence of functions $\left\{A_{n}(t)\right\}$, and where, moreover, there are no zeros of the function $\Delta_{k}(t)$ on $\gamma$.

Remarks. (i) Curve $\gamma$ need not be a circle; it suffices that $\gamma$ be a rectifiable simple closed curve surrounding $t=0$ and lying in the ring $\delta<|t|<R^{\prime}$ where $R^{\prime}$ is the minimum radius of convergence of the power series $\left\{A_{n}(t)\right\}$ (defined by (1.2)).

(ii) Let $R^{\prime \prime}$ be chosen (not to exceed $R^{\prime}$ ) so that $\Delta_{k}(t)$ has no zeros in the ring $\delta<|t|<R^{\prime \prime}$. Then all contours $\gamma$, of Remark (i), that lie in this new ring will obviously yield the same solution (2.16). Inspection of (2.14) shows that the type of $\left\{y_{n}\right\}$ and hence of $\left\{x_{n}\right\}$ cannot exceed $d$, where $d$ is the maximum distance from $\gamma$ to the origin. Since $\gamma$ can be chosen as close to the circle $|t|=\delta$ as we wish, it follows that for $\gamma$ in the ring $\delta<|t|<R^{\prime \prime}$, the type of the solution (2.16) cannot exceed $\delta$. If $\left(\left(x_{n}\right)\right)<\delta$, it is easy to show that the sequence $A_{n}\left[\left\{x_{n}\right\}\right]$, that is, the sequence $\left\{c_{n}\right\}$, is also of type less than $\delta$; which is a contradiction. Hence we conclude that if contour $\gamma$ lies in the ring $\delta<|t|<R^{\prime \prime}$, the solution (2.16) is of type equal to $\delta$.

Corollary. The solution (2.16) can be written

$$
x_{n}=\frac{k}{2 \pi i} \int_{\gamma} \frac{t^{n-1}}{\Delta_{k}(t)} \cdot \Delta_{k}(t ; H) d t,
$$

where $\Delta_{k}(t ; H)$ is given by (1.6).

This follows at once from (1.6), (2.14) and the meaning of $\Delta_{k ; 0, j}(t)$

3. Proof of the theorem. We now establish Theorem 1.1. Choose the number $Q$ to be less than $q$ and also to lie in the range $\delta<Q \leqq q_{2}$. Consider an arbitrary positive integer $k$, and denote by $\mathcal{A}^{(k)}$ the $k$-periodic system obtained from (1.1) by using the first $k$ equations of (1.1); that is, systems (1.1) and $\mathcal{A}^{(k)}$ agree in their first $k$ equations. All the results of $\S 2$ apply to $\mathcal{A}^{(k)}$ if we use $Q$ in place of $q$.

Since (1.9) holds uniformly (in the ring $R$ ), there exists an integer $S$ such that if for an $s>S$ the denominator $\Delta_{k s}(t)$ has zeros in the ring, then these zeros are completely cancelled by corresponding 
zeros in the numerator $\Delta_{k s}(t ; H)$. It is therefore not necessary that the contour $\gamma$ avoid zeros ${ }^{4}$ of the denominator.

Choose $\gamma$ as a circle whose radius $r$ lies in the range $\delta<r<Q$. Given $\epsilon>0$, there exists an $S=S_{\epsilon}$ such that for all $s>S$,

$$
\left|\left\{k_{s} \Delta_{k_{s}}(t ; H) / \Delta_{k_{s}}(t)\right\}-\Delta^{*}(t)\right|<\epsilon,
$$

uniformly on $\gamma$. Let $\left\{x_{n}^{\left(k_{s}\right)}\right\}$ be the solution given by (2.17) corresponding to the $k_{s}$-periodic system $\mathcal{A}^{\left(k_{s}\right)}$, and let $\left\{x_{n}\right\}$ be given by (1.10). Then

$$
\begin{aligned}
\left|A_{p}\left[\left\{x_{n}^{\left(k_{s}\right)}\right\}\right]-A_{p}\left[\left\{x_{n}\right\}\right]\right| \equiv\left|\sum_{n=0}^{\infty} a_{p n} x_{n+p}^{\left(k_{s}\right)}-\sum_{n=0}^{\infty} a_{p n} x_{n+p}\right| \\
=\left|\frac{1}{2 \pi i} \int_{\gamma} t^{p-1} A_{p}(t)\left[\left\{k_{s} \Delta_{k_{s}}(t ; H) / \Delta_{k_{s}}(t)\right\}-\Delta^{*}(t)\right] d t\right| \\
\leqq M_{p} \cdot \boldsymbol{r}^{p} \cdot \boldsymbol{\epsilon},
\end{aligned}
$$

where $M_{p}=\max \left|A_{p}(t)\right|$ for $t$ on $\gamma$.

Let $p$ be arbitrary but fixed, and choose $s$ (already subject to the condition $s>S$ ) so that $k_{s}>p$. Then

$$
A_{p}\left[\left\{x_{n}^{\left(k_{s}\right)}\right\}\right]=c_{p}
$$

since $\left\{x_{n}^{\left(k_{s}\right)}\right\}$ satisfies system $\mathcal{A}^{\left(k_{s}\right)}$. Hence

$$
\left|c_{p}-A_{p}\left[\left\{x_{n}\right\}\right]\right|<M_{p} \cdot r^{p} \cdot \epsilon .
$$

Since $p$ is fixed and $\epsilon$ is arbitrary, it follows that

$$
A_{p}\left[\left\{x_{n}\right\}\right]=c_{p} .
$$

That is, $\left\{x_{n}\right\}$ satisfies the $p$ th equation of system (1.1); and from the arbitrariness of $p,\left\{x_{n}\right\}$ satisfies system (1.1). The portion of Theorem 1.1 referring to the type of solution $\left\{x_{n}\right\}$ follows at once from (1.10), so the proof of Theorem 1.1 is complete.

Remarks. (i) The hypothesis of Theorem 1.1 can be weakened in the following way without affecting the conclusion: Replace the condition that (1.9) holds uniformly in the ring $R: q_{1}<|t|<q_{2}$ with $\delta<q_{2} \leqq q$ by the condition that (1.9) holds uniformly on $\gamma$, where $\gamma$ is a rectifiable simple closed curve surrounding the origin and lying in the ring $\delta<|t|<q$.

(ii) If $G(t)$ is analytic in and on $\gamma$, then $(1 / 2 \pi i) \int_{\gamma} t^{n} G(t) d t=0$,

${ }^{4}$ Since each $\Delta_{k}(t)$ has in $|t| \leqq Q$ only a finite number of zeros, it is possible to choose $\gamma$ in the ring $R$ so as to avoid all such zeros. For the purpose of a later Remark however, it is undesirable that $\gamma$ be so restricted. 
$n=0,1, \cdots$. In the ratio $\Delta_{k}(t ; H) / \Delta_{k}(t)$ that occurs in (1.9), one may therefore throw away all the positive powers of $t$ (in the Laurent expansion for the ratio). Or one may state more generally: If there exists a sequence of functions $\left\{G_{k}(t)\right\}$, analytic in and on $\gamma$, and a sequence $\left\{k_{s}\right\}$, such that

$$
\lim _{s=\infty}\left[\left\{k_{s} \Delta_{k_{s}}(t ; H) / \Delta_{k_{s}}(t)\right\}+t G_{k_{s}}(t)\right]=\Delta^{* *}(t),
$$

the convergence being uniform on $\gamma$, then (1.1) has the solution

$$
x_{n}^{* *}=\frac{1}{2 \pi i} \int_{\gamma} t^{n-1} \Delta^{* *}(t) d t .
$$

4. $k$-periodic system, homogeneous case. It will now be shown that the homogeneous $k$-periodic system also has solutions that can be exhibited in closed form. The homogeneous system is

$$
a_{n 0} x_{n}+a_{n 1} x_{n+1}+\cdots=0 \quad(n=0,1, \cdots) .
$$

It is assumed as before that $A_{n}(t)$ is analytic in $|t| \leqq q$ and that $A_{n}(0) \neq 0$. Since the determinant $\Delta_{k}(t)$ of $(1.8)$ has the property (2.5), it follows that the zeros (if any) of $\Delta_{k}(t)$ in $|t| \leqq q$ come in nests of $k$ zeros each, such that if $\alpha$ is a zero, then the zeros in the same nest $t^{5}$ are $\omega_{k}^{s} \alpha, s=0,1, \cdots, k-1$. Moreover all zeros of a nest have the same multiplicity, so that the total number $m$ of zeros of $\Delta_{k}(t)$ in $|t| \leqq q$ is a multiple of $k: m=l k$. We have termed $l$ the order ${ }^{6}$ of $\Delta_{k}(t)$ (in $|t| \leqq q)$, and have shown ${ }^{7}$ that system (4.1) has precisely $l$ linearly independent solutions of type not exceeding $q$.

Consider a fixed nest of zeros $\left\{\omega_{k}^{s} \alpha\right\}$. Let $C$ be a rectifiable simple closed curve ${ }^{8}$ surrounding the origin and all the zeros of the nest, with all the other zeros of $\Delta_{k}(t)$ exterior to $C$; and define the sequences $X_{j, l}^{(r)}:\left\{x_{j l ; n}^{(r)}\right\}$ by

$$
x_{j l ; n}^{(r)}=\frac{1}{2 \pi i} \int_{C} \frac{t^{n+l}\left(t^{k}-\alpha^{k}\right)^{r}}{\Delta_{k}(t)} \cdot \Delta_{k ; 0, j}(t) d t,
$$

where $j, l$ are in the range $0,1, \cdots, k-1$, and $r$ is a non-negative integer. The value of this integral is the same for all curves $C$ fulfilling the above conditions. It is therefore no restriction to suppose,

\footnotetext{
${ }^{5}$ Note their symmetric position about the origin.

${ }^{6}$ Tr., $\$ 2$ loc. cit.

${ }^{7}$ Tr., Theorem 3.6, loc. cit.

${ }^{8} C$ is also to lie in the common circle of convergence of the functions $A_{n}(t)$, $n=0,1, \cdots, k-1$.
} 
as we now do, that $C$ is chosen so as to be invariant under a rotation of angle $2 \pi / k$ around the origin.

Let $p=0,1, \cdots, k-1 ; s=0,1, \cdots$ Then, using (2.2),

$$
\begin{aligned}
A_{k s+p}\left[X_{j, l}^{(r)}\right] & \equiv \sum_{n=0}^{\infty} a_{p n} x_{j l ; k s+p+n}^{(r)} \\
& =\frac{1}{2 \pi i} \int_{C} \frac{t^{k s+p+l}\left(t^{k}-\alpha^{k}\right)^{r}}{\Delta_{k}(t)} \cdot A_{p}(t) \Delta_{k ; 0, j}(t) d t .
\end{aligned}
$$

Since the only singularities of the integrand interior to $C$ are at the zeros $\left\{\omega_{k}^{s} \alpha\right\}$, we can replace $C$ by $k$ contours $C_{0}, \cdots, C_{k-1}$, where $C_{\lambda}$ surrounds only the zero $\omega_{k}^{\lambda} \alpha$. In fact, $C_{\lambda}$ may be chosen as a small circle centered at this zero, and if the radii of these circles are equal, then a rotation of angle $2 \pi / k$ around the origin will carry each circle into the next one.

This replacement of $\int_{C}$ by $\sum_{\lambda=0}^{k-1} \int_{C_{\lambda}}$ being accomplished, make the change of variable $t=\omega_{k}^{\lambda} u$ in the integral $\int c_{\lambda}$. As $t$ traces $C_{\lambda}$ the variable $u$ traces $C_{0}$. Consequently,

$$
\begin{aligned}
A_{k s+p}\left[X_{i, l}^{(r)}\right]= & \frac{1}{2 \pi i} \int_{C_{0}} \frac{\left(u^{k}-\alpha^{k}\right)^{r}}{\Delta_{k}(u)} \\
& \cdot u^{k s+p+l}\left\{\sum_{\lambda=0}^{k-1} \omega_{k}^{\lambda(p+l+1)} A_{p}\left(\omega_{k}^{\lambda} u\right) \Delta_{k ; 0, j}\left(\omega_{k}^{\lambda} u\right)\right\} d u ;
\end{aligned}
$$

and on using (2.9),

$$
A_{k s+p}\left[X_{j, l}^{(r)}\right]=\frac{1}{2 \pi i} \int_{C_{0}} \frac{\left(u^{k}-\alpha^{k}\right)^{r}}{\Delta_{k}(u)} \cdot u^{k s+p+l} \cdot \Phi_{p, l, j}(u) d u,
$$

where

$$
\Phi_{p, l, j}(u)=\sum_{\lambda=0}^{k-1} \omega_{k}^{\lambda(p+l+j+1)} A_{p}\left(\stackrel{\lambda}{\omega}_{k} u\right) \Delta_{k ; \lambda, j}(u) d u .
$$

For each $j$, choose $l=k-j-1$. Then from (2.11) we obtain the relation

$$
\Phi_{p, k-j-1, j}(u)=\left\{\begin{array}{ccc}
\Delta_{k}(u) & \text { if } & p=j, \\
0 & \text { if } & p \neq j .
\end{array}\right.
$$

In both cases (4.5) becomes

$$
A_{k s+p}\left[X_{j, k-j-1}^{(r)}\right]=0 .
$$

To sum up:

THEOREM 4.1. Let the functions $\left\{A_{n}(t)\right\}$ be analytic in $|t| \leqq q$, with 
$A_{n}(0) \neq 0$. If $\left\{\omega_{k}^{s} \alpha\right\}, s=0,1, \cdots, k-1$, is a nest of zeros of $\Delta_{k}(t)$ with $|\alpha| \leqq q$, then for each $j=0,1, \cdots, k-1$ and for each $r=0,1, \cdots$, the sequence (4.2) with $l=k-j-1$ is a solution of the $k$-periodic homogeneous system (4.1).

In Theorem 4.1 it is conceivable that one or more of the solutions (4.2), with $l=k-j-1$, is identically zero. (This is certainly true for $r$ sufficiently large.) We therefore examine the problem of linear independence. On eliminating $l$ in (4.2) we obtain sequences to be denoted by $X_{j}^{(r)}$ :

$$
X_{j}^{(r)}: \quad x_{j, n}^{(r)}=\frac{1}{2 \pi i} \int_{C} \frac{t^{n+k-j-1}\left(t^{k}-\alpha^{k}\right)^{r}}{\Delta_{k}(t)} \cdot \Delta_{k ; 0, j}(t) d t ;
$$

and these are the sequences that have been shown to satisfy $y^{9}(4.1)$.

If we replace $C$ by its equivalent, namely the sum of the contours $C_{0}, \cdots, C_{k-1}$, and on $C_{p}$ set $t=\omega_{k}^{p} u$, then (4.9) is seen to become

$$
x_{j, n}^{(r)}=\frac{1}{2 \pi i} \int_{C_{0}} \frac{\left(u^{k}-\alpha^{k}\right)^{r}}{\Delta_{k}(u)} \cdot u^{n+k-j-1} \cdot \Lambda_{k ; j, n}(u) d u,
$$

where

$$
\Lambda_{k ; j, n}(u)=\sum_{p=0}^{k-1} \omega_{k}^{p n} \Delta_{k ; p, j}(u)
$$

It is clear that

$$
\Lambda_{k ; j, n+8 k}(u)=\Lambda_{k ; j, n}(u) .
$$

Also, by use of (2.9), one obtains the relation $\Delta_{k ; p, j}\left(\omega_{k} u\right)=\omega_{k}^{j} \Delta_{k ; p+1, j}(u)$. Applying this to (4.11) one readily shows that

$$
\Lambda_{k ; j, n}\left(\omega_{k} u\right)=\omega_{k}^{j-n} \Lambda_{k ; j, n}(u) .
$$

From (4.13) it follows that

$$
\left(\omega_{k} u\right)^{n+k-j-1} \Lambda_{k ; j, n}\left(\omega_{k} u\right)=\frac{1}{\omega_{k}}\left[u^{n+k-j-1} \Lambda_{k ; j, n}(u)\right],
$$

so that the bracket possesses a power series of the following type:

$$
u^{n+k-i-1} \Lambda_{k ; j, n}(u)=\sum_{s=1}^{\infty} b_{j, n ; s-1} u^{s k-1} ;
$$

${ }^{9}$ They are also of type not exceeding $q$. 
and from (4.12),

$$
u^{n+p k-j-1} \Lambda_{k ; j, n+p k}(u)=u^{p k} \sum_{s=1}^{\infty} b_{j, n ; s-1} u^{s k-1} .
$$

Suppose $\alpha$ is a zero of multiplicity $h$ for $\Delta_{k}(t)$. Then this is also the multiplicity of each of the zeros in the nest $\left\{\omega_{k}^{s} \alpha\right\}$, so that

$$
\Delta_{k}(t)=\left(t^{k}-\alpha^{k}\right)^{h} \cdot Q_{k}(t)
$$

where $Q_{k}(t)$ is analytic in $|t| \leqq q+\epsilon(\epsilon>0$ sufficiently small) and has the properties $Q_{k}\left(\omega_{k}^{s} \alpha\right) \neq 0$ and $Q_{k}\left(\omega_{k} t\right)=Q_{k}(t)$.

Let the circles $C_{0}, \cdots, C_{k-1}$ be chosen so small that as $u$ traces $C_{0}$, then $z=u^{k}$ traces exactly once a rectifiable simple closed curve $T_{0}$ that surrounds $z=\alpha^{k}$ and has $z=0$ in its exterior. We have

$$
\Delta_{k}(u)=\left(z-\alpha^{k}\right)^{h} \cdot q_{k}(z)
$$

where $q_{k}(z)$ is analytic in and on $T_{0}$ and nowhere zero there. Consequently

$$
x_{j, n}^{(r)}=\frac{1}{2 k \pi i} \int_{T_{0}}\left(z-\alpha^{k}\right)^{r-h} \cdot D_{k ; i, n}(z) d z,
$$

where

$$
D_{k ; j, n}(z)=\frac{1}{q_{k}(z)} \cdot \sum_{s=0}^{\infty} b_{j, n ; s} z^{s}=\sum_{s=0}^{\infty} d_{j, n ; s}\left(z-\alpha^{k}\right)^{s} .
$$

If a $j$ and a $p$ exist in the range $0,1, \cdots, k-1$ such that

$$
D_{k ; j, p}\left(\alpha^{k}\right) \neq 0 \text {, }
$$

then the $h$ sequences (4.9), for $r=0,1, \cdots, h-1$, constitute a linearly independent set ${ }^{10}$ of solutions of (4.1). To see this, we first observe that from (4.15) and (4.16) follows the relation $D_{k ; j, m+s k}(z)$ $=z^{8} D_{k ; j, m}(z)$, so that

$$
x_{j, m+\delta k}^{(r)}=\frac{1}{2 k \pi i} \int_{T_{0}}\left(z-\alpha^{k}\right)^{r-h} \cdot z^{8} D_{k ; j, m}(z) d z .
$$

Hence if $X_{j}^{(0)}, \cdots, X_{j}^{(h-1)}$ are linearly dependent, then constants $\lambda_{r}$ exist, not all zero, such that

$$
\{0\}=\sum_{r=0}^{h-1} \lambda_{r} X_{j}^{(r)} ;
$$

${ }^{10}$ If $r \geqq h,(4.18)$ shows that $\left\{x_{j, n}^{(r)}\right\} \equiv\{0\}$. 
so, setting $n=m+s k$,

$$
0=\sum_{r=0}^{h-1} \lambda_{r} x_{j, n}^{(r)}=\frac{1}{2 k \pi i} \int_{T_{0}} z^{s} D_{k ; i, m}(z)\left\{\sum_{r=0}^{h-1} \lambda_{r}\left(z-\alpha^{k}\right)^{r-h}\right\} d z
$$

This is an identity for $m=0,1, \cdots, k-1$ and for all $s=0,1, \cdots$. Choose $s=0,1, \cdots, h-1$ and multiply the $s$ th relation by $\mu_{s}$ where $\mu_{s}$ is chosen so that

$$
\sum_{s=0}^{h-1} \mu_{s} z^{s}=\left(z-\alpha^{k}\right)^{h-1}
$$

Also take $m=p$. Then

$$
0=\lambda_{0} \int_{T_{0}} D_{k ; j, p}(z)\left(z-\alpha^{k}\right)^{-1} d z=\lambda_{0} D_{k ; j, p}\left(\alpha^{k}\right) ;
$$

and by assumption (4.20), we must therefore have $\lambda_{0}=0$. In the same way it is shown that $\lambda_{1}=\cdots=\lambda_{h-1}=0$. This contradiction establishes the linear independence of $X_{j}^{(0)}, \cdots, X_{j}^{(h-1)}$, as was asserted.

From (4.15) and (4.19) we see that condition (4.20) is equivalent to this: $p$ and $j$ exist in $0,1, \cdots, k-1$ such that ${ }^{11}$

$$
\Lambda_{k ; j, p}\left(\dot{\omega}_{k}^{*} \alpha\right) \neq 0, \quad s=0,1, \cdots, k-1 .
$$

Now suppose that (4.20), and hence (4.23), does not hold. Then for every $j, p, s$ in $0,1, \cdots, k-1$ we shall have

$$
\Lambda_{k ; j, p}\left(\stackrel{8}{\omega_{k} \alpha} \alpha\right)=0 \text {. }
$$

For each fixed $j$ and $s$, take $u=\omega_{k}^{s} \alpha$ in (4.11). We obtain a system of linear homogeneous equations with a nonvanishing Vandermond determinant, so the "unknowns" must all be zero. Thus,

$$
\Delta_{k ; p, j}\left(\omega_{k}^{\circ} \alpha\right)=0 \quad(p, j, s=0,1, \cdots, k-1) .
$$

Conversely, (a) follows from (b).

If we agree to say that system (4.1) is nonsingular at the nest of zeros $\left\{\omega_{\boldsymbol{k}}^{s} \alpha\right\}$ if there exists a triple of integers $(p, j, s)$ in the range $0,1, \cdots, k-1$ such that

$$
\Delta_{k ; p, j}\left(\stackrel{\bullet}{\omega_{k} \alpha} \alpha\right) \neq 0,
$$

then we have established the following result:

${ }^{11}$ If (4.23) holds for one value of $s$ it will then hold for all $s$, as we see from (4.12). 
THEOREM 4.2. In the k-periodic homogeneous system (4.1) let the functions $A_{n}(t)$ be analytic in $|t| \leqq q$, with $A_{n}(0) \neq 0$. If (4.1) is nonsingular at the nest of zeros $\left\{\omega_{k}^{s} \alpha\right\}, s=0,1, \cdots, k-1$, then the $h$ sequences $X_{j}^{(0)}, \cdots, X_{j}^{(h-1)}$ of (4.9) are linearly independent solutions of system (4.1) and are of type not exceeding $q$.

Remarks. (i) Let the order of $\Delta_{k}(t)$ in $|t| \leqq q$ be $l$. If system (4.1) is nonsingular at every nest of zeros, then Theorem 4.2 provides us with sets of solutions, the total number of such solutions being exactly $l$, and the solutions in each set are linearly independent. It can be shown (although we shall not do so here) that the totality of these $l$ solutions are linearly independent, and that they therefore constitute a complete set of solutions of type not exceeding $q$, of (4.1).

(ii) If system (4.1) is not nonsingular at some nest of zeros $\left\{\omega_{k}^{s} \alpha\right\}$, then (4.9) will not yield $h$ linearly independent solutions, but may yield some. There are $h$ linearly independent solutions corresponding to this nest, but their representation is not so simple as (4.9), and we do not discuss them here.

(iii) One can proceed from (4.9) by a limiting process, and arrive at a result analogous to Theorem $\mathbf{1 . 1}$ for the homogeneous system corresponding to (1.1). However, one cannot be certain that the solution so obtained is not actually $x_{n} \equiv 0$.

The Pennsylvania State College 